\title{
Examination of an Extension of Guttman's Model of Ability Tests
}

\author{
Aharom Tziner. \\ Tel-Aviv Universien \\ Avigdor Rimmer, \\ Pilat, Lid. Tel-Aviy
}

\begin{abstract}
An extension of Guttman's structural model of ability tests was devised and investigated with two samples consisting, respectively, of 335 and 225 males. The examinees in the first sample came for vocational guidance after their military service and were administered a 17-test battery. The second sample consisted of applicants for various jobs in an organization and were administered a 14-test battery. For each sample, a matrix of intercorrelations between scores was obtained based on the number of correct responses. The matrices were submitted to Guttman-Lingoes Smallest Space Analysis. The two-dimensional structure found was a radex in which (1) the facet of the language of presentation radially divided the space and (2) the facet of mental operation formed concentric rings. The significance of these findings for theoretical and applied problems relating to ability tests is discussed.
\end{abstract}

The underlying structure of ability tests is a topic of considerable interest (cf., Cunningham, 1981; Kelderman, Mellenbergh, \& Elshout, 1981). There appear to be two primary reasons for this. First, from a general perspective of ability testing it is important to have a coherent structural model that reasonably organizes the tests into meaningful facets. Such a model could aid in deciding, in a systematic and rational manner, what new tests should be developed in order to measure facets previously only partially assessed, or not assessed at all. Second, researchers are interested in determining

APPLIED PSYCHOLOGICAL MEASUREMENT

Vol. 8, No. 1, Winter 1984, pp. 59-69

(C) Copyright 1984 Applied Psychological Measurement Inc.

0146-6216/84/010059-11\$1.80 whether there is a universal structural model of general applicability that does not vary even when used for different testing objectives (e.g., vocational counseling or selection for job openings in organizations) or when used on individuals with varying levels of education, age, and so forth.

Previous studies that were aimed at examining the foregoing questions were mainly based on factor analysis (see, for instance, Guilford, 1980, 1981). This method, however, has been sharply criticized on several grounds. Basic assumptions of its statistical model, as, for instance, linearity, are often violated (Kelderman et al., 1981). Apart from this, factors with only one or two salient loadings are frequently found when using factor analysis to reveal the structure of ability tests (Cunningham, 1981). In such a context, the decision regarding the appropriate number of factors becomes unusually difficult, thus leading to solutions that are not parsimonious and giving rise to an inconsistency across investigators (Schlesinger \& Guttman, 1969). Furthermore, although the factor analytic strategy of research has often been used to test hypotheses of structure, it has also been shown to be inadequate for this purpose (Guttman, 1958; Shapira \& Zevulun, 1979; Shye, 1978).

A better approach to the structural configuration issue might emerge from Guttman's facet theory (for a detailed exposition see Shirom, 1982; Shye, 1978). Relying on this approach, Schlesinger and Guttman (1969) classified ability tests into two fac- 
ets: (1) the language of presentation of the test items, which may be verbal, figural, or numerical; and (2) the mental operation required by the test, namely, rule inference or rule application. They then defined a mapping sentence to corroborate the structural configuration based on the foregoing facets. The empirical investigation, using high school students as subjects, firmly supported the hypothesized structural configuration: a radex yielded by a radial arrangement of tests according to the language of presentation facet, together with a pattern of concentric circles formed by the mental operation facet.

Nevertheless, it is important to outline a weakness inherent in one of the facets - that of mental operation. This facet did not take into account the important differentiation in mental operations indicated by Piaget (1956; and also in Brainerd, 1978). According to Piaget, the rule of application operation entails three levels of cognitive complexity organized hierarchically from the lowest to the highest:

1. Ability to successfully handle concrete items (designated here concrete rule application),

2. Ability to successfully handle signs (clerical rule application), and

3. Ability to successfully handle cognitive concepts (abstract-cognitive rule of application).

For this reason the mental operation was resiructured in the present study so that it would be composed of four elements instead of two:

1. Rule inference,

2. Abstract-cognitive rule application,

3. Clerical rule application, and

4. Concrete rule application.

Using this extension, a mapping sentence was devised similar to that formulated and examined by Schlesinger and Guttman (1969) in order to extract the maximum from the operation facet. The aim was to find out empirically whether or not the radex structural configuration is still preserved after the introduction of the above-mentioned extension. Two replications were carried out: one on a sample of individuals applying for vocational counseling, the other on a sample of selected applicants for job positions in organizations. It was then decided to test the mapping sentence, employing two replications, in order to ascertain whether the objectives of testing in any way affected the structural configuration of ability tests that emerged.

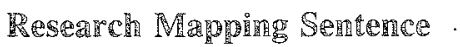

The mapping sentence tested in the current study is shown in Figure 1 . The aim was to examine empirically whether the radex would still be an appropriate structural configuration to portray geometrically the interrelationships among the elements of facets, even when using this elaborate mapping sentence.

\section{Method}

\section{Subjects and Proced}

The first sample consisted of 335 males, mostly aged 21 to 22 , selected at random from applicants for vocational guidance after completion of their military service. The second sample consisted of 225 males aged 21 to 35, also selected at random, from applicants for job positions in an organization. Both groups completed ability tests that were administered and scored by professional psychologists-17 tests for those in the first sample and 14. for those in the second. Examinees in the first sample were tested at the Vocational Branch of the Israell Ministry of Labor and Welfare in Tel Aviv, and those in the second sample were tested at a private psychological testing company providing selection and placement services.

\section{V习rian}

The combination of elements of the two facets, $\mathrm{A}$ and $\mathrm{B}$, forms profiles (structuples in facet theory terminology) represented here by the ability tests. For instance, the Verbal Analogies test represents the $a_{1} b_{1}$ structuple. The detailed list of tests employed, along with the structuples to which they correspond, is presented in Table 1.

\section{Data $\mathbb{A}$ mallysis $\mathbb{P}$ rocedure}

The investigation of the empirical structure of the ability tests was carried out using Guttman- 
Figure 1

The Mapping Sentence for Ability Tests

The performance of subject $|x|$ on a psychometric ablity lest isem presented in

\section{Facet A: Language Facet B: Mental Operation}

of Presentation

$\left\{\begin{array}{l}a_{2} \text { Numerical } \\ a_{3} \text { Figural }\end{array}\right\} \quad\left\{\begin{array}{c}b_{1} \text { Pule inference } \\ b_{2} \text { Abstract cognisive } \\ \text { rule application } \\ b_{3} \text { Clerical rule application } \\ b_{4} \text { Concrete rule application }\end{array}\right\} \rightarrow$

$\rightarrow\left\{\begin{array}{c}\text { High } \\ : \\ : \\ : \\ \text { Low }\end{array}\right\}$

Lingoes Smallest Space Analysis (SSA; see, e.g., Guttman, 1968; Lingoes, 1973). This is a nonmetric multidimensional scaling procedure that geometrically represents the correlation matrix based on the order of magnitude of the intercorrelations among the variables - in this case, the tests. SSA portrays the tests in the current study as points in an Euclidean space. The more the correlation between the corresponding tests increases, the nearer the two points are to each other. More explicitly, if $r_{i j}$ is the observed correlation between tests $i$ and $j$, and if $d_{i j}$ is the calculated distance between these two tests for the smallest space, then SSA attempts to find out the space with the minimal number of dimensions in which the following inequalities are preserved:

$d_{i j}<d_{k l}$ whenever $r_{i j}>r_{k l}$

$$
(i, j, k, l=1,2, \ldots, n)
$$

Order among the correlations is thus preserved in the smallest possible Euclidean space, and the absolute sizes of the input correlation coefficients can be reproduced from the scattergram of the $r_{i j}$ on the $d_{i j}$ (Shepard diagram) printed by the computer. Such a chart indicates how well $r_{i j}=f\left(d_{i j}\right)$, where $f$ is a monotonically decreasing function.

The goodness of fit between the smallest possible Euclidean space and the correlation matrix is numerically measured by a coefficient of alienation $\left(1-r^{2}\right)^{1 / 2}$, where $r$ is a rank-order correlation between the intercorrelations of the tests and their corresponding geometric distances. The smaller the coefficient of alienation, the better the fit. Zero represents a perfect fit. In practice, however, a coefficient of alienation smaller than .15 is considered a good fit (see Guttman, 1968).

To find evidence regarding the structural configuration in the SSA output, it is necessary to apply the principles of contiguity regions (Elizur, 1981; Shapira \& Zevulun, 1978). In this comnection, and 


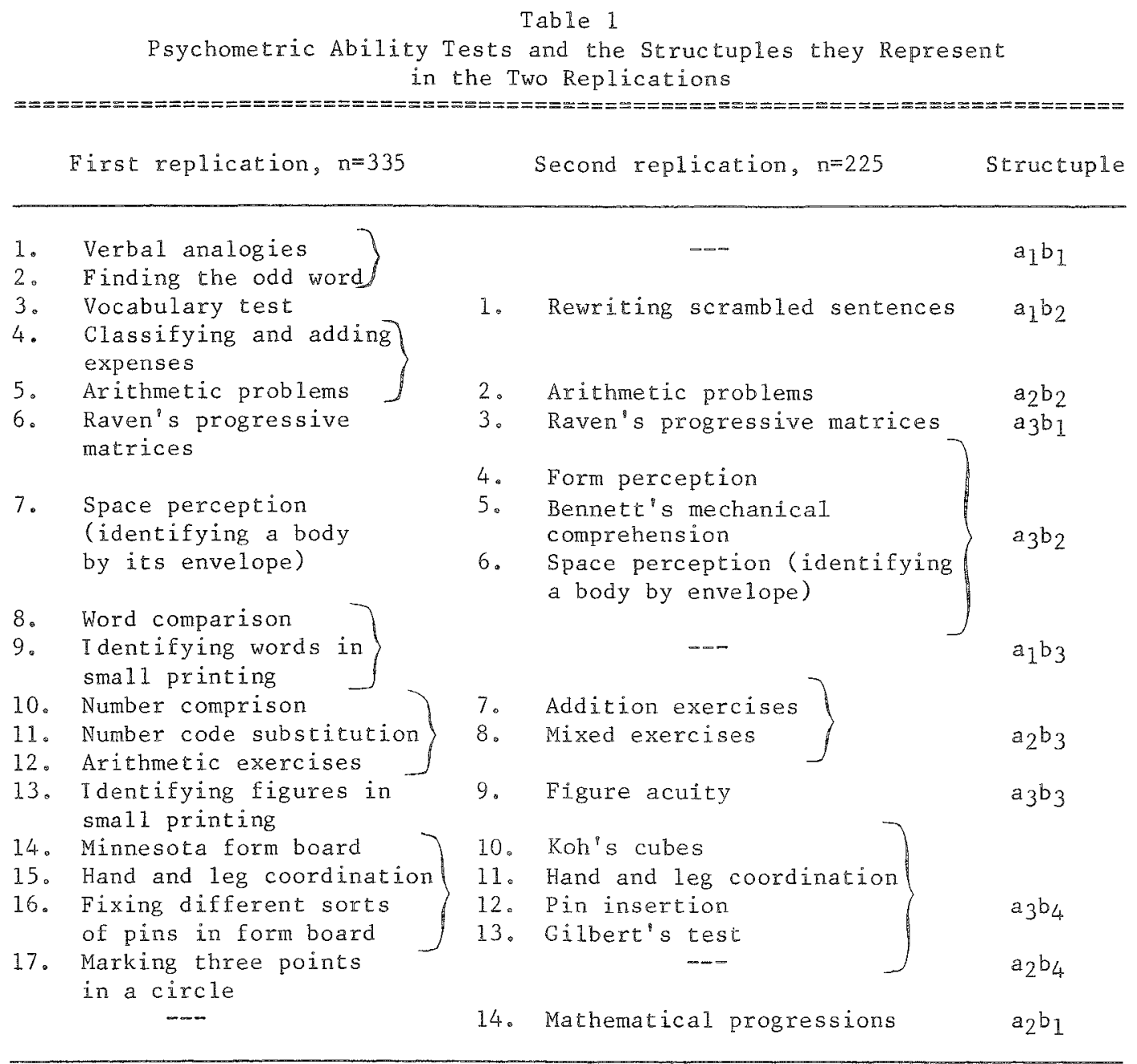

in order to detect a radex structure, one of the facets in the mapping sentence of this study (A) should have acted as a polarizing facet, while the other (B) should have acted as a modulating facet. A facet is designated as modulating when it divides the space into concentric circles and as polarizing when three or more lines originating at the same point (or pole) separate the space by diverging into different directions (Schlesinger \& Guttman, 1969). Additional relevant details pertaining to the principles of contiguity in facet analysis can be found elsewhere (Gratch, 1973; Shye, 1978).

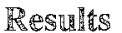

First computed among the number of correct responses to the tests were Pearson intercorrelations. Tables 2 and 3 display the results for the first and second replication, respectively. The first point of interest that can be seen in both tables is that all the correlations are zero or positive, fulfilling one of the requirements set up by Gutman for facet analysis (for more details refer to Gratch, 1973). In addition, the correlations in the first application are much higher than in the second application, a 


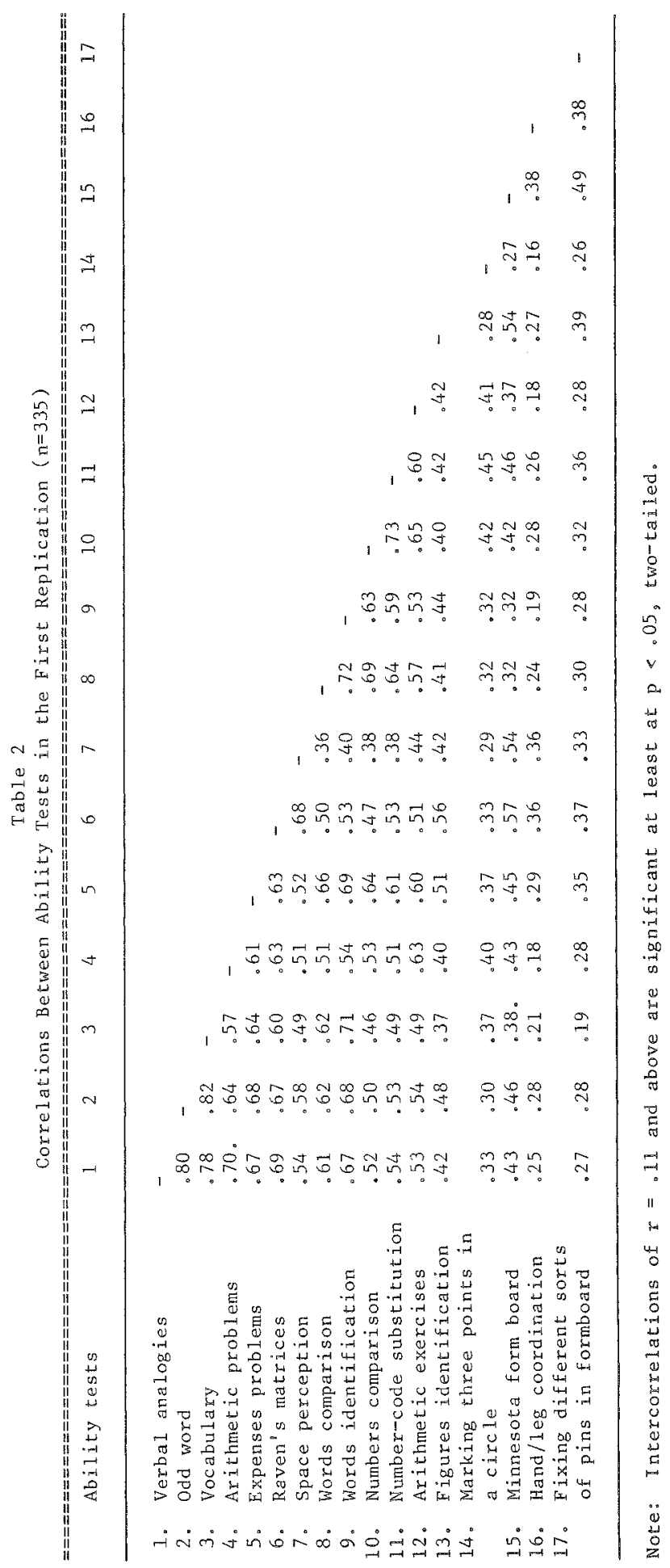

Downloaded from the Digital Conservancy at the University of Minnesota, http://purl.umn.edu/93227. May be reproduced with no cost by students and faculty for academic use. Non-academic reproduction requires payment of royalties through the Copyright Clearance Center, http://www.copyright.com/ 


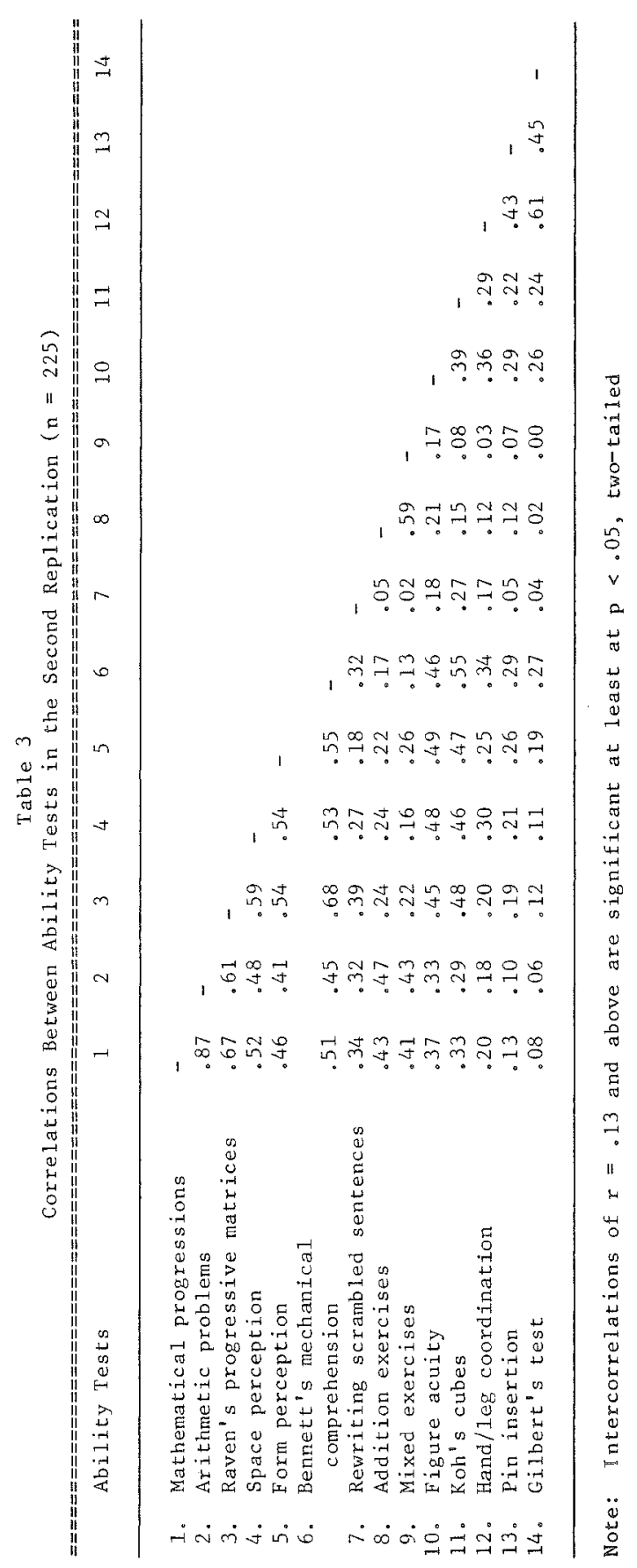

Downloaded from the Digital Conservancy at the University of Minnesota, http://purl.umn.edu/93227. May be reproduced with no cost by students and faculty for academic use. Non-academic reproduction requires payment of royalties through the Copyright Clearance Center, http://www.copyright.com/ 
fact that can be explained by the first sample being more heterogeneous than the second, as dictated by the objective of testing (vocational guidance versus selection and placement for a particular organization).

These matrices were then subjected to the SSAI computer program, which maps the tests as points on the Euclidean space of two dimensions. These test configurations are shown in Figures 2 and 3 , corresponding to the matrices in Tables 2 and 3 , respectively. The coefficients of alienation obtained were 11 and .095 , respectively, for the configurations in Figures 2 and 3, which can be con- sidered as indicating good and very good fit of the two-dimensional plots to the original intercorrelation matrices (as references for goodness of fit, see Gutman, 1968; Kenny \& Canter, 1981). Both figures show that every test is contiguous with others that share the same facet elements. This is consistent with the principle of contiguity in facet theory (see Shye, 1978), which explicitly states that items having the same facet elements should be more highly correlated and thus closer together in the configurative plot than items that do not. This is illustrated here, for example, by Verbal Analysis and Finding-the-Odd-Word tests pertaining to the

Fige 2

SSA Map for the 17 Tests in the First Group

(Vocational Guidance Situation)

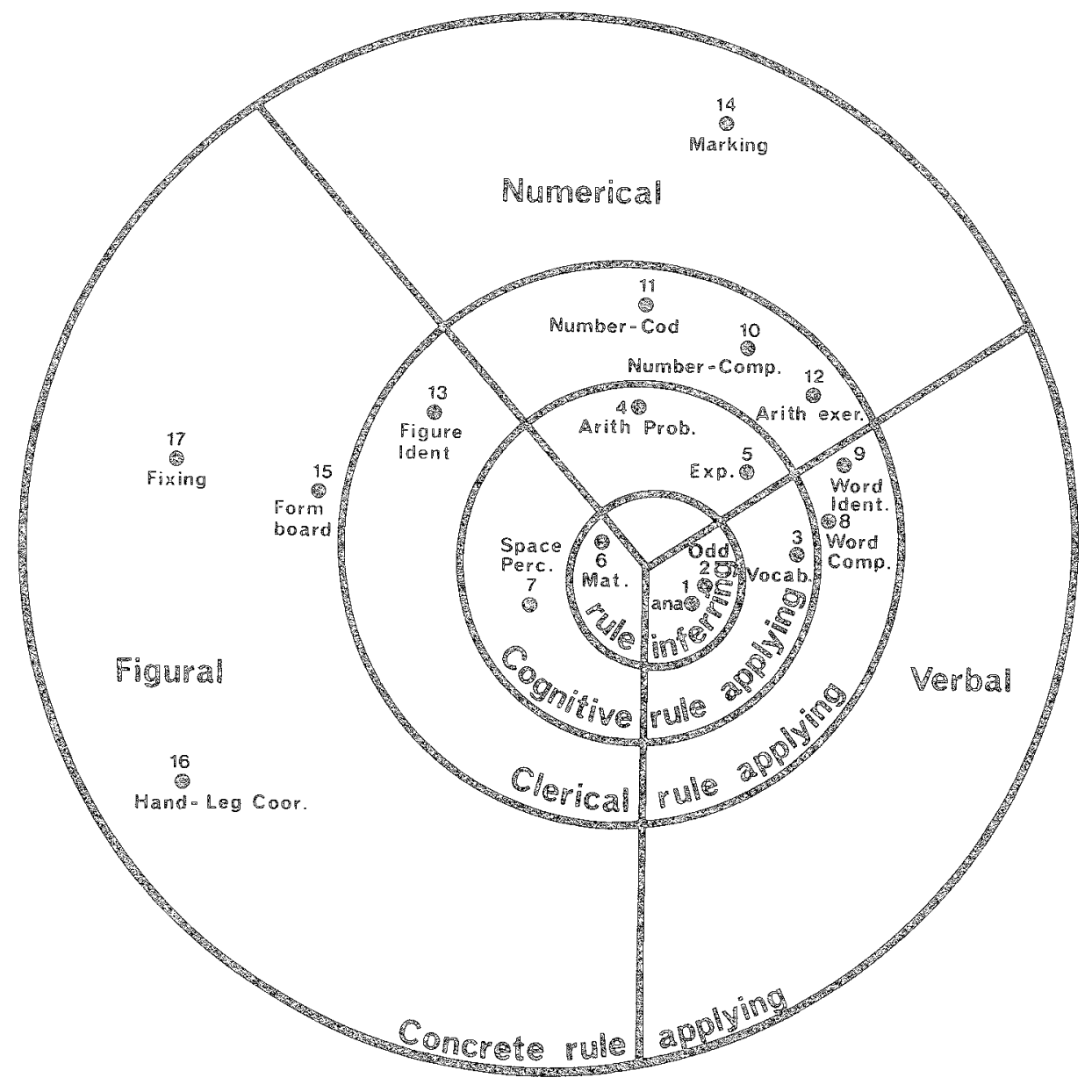

Downloaded from the Digital Conservancy at the University of Minnesota, http://purl.umn.edu/93227. May be reproduced with no cost by students and faculty for academic use. Non-academic reproduction requires payment of royalties through the Copyright Clearance Center, http://www.copyright.com/ 


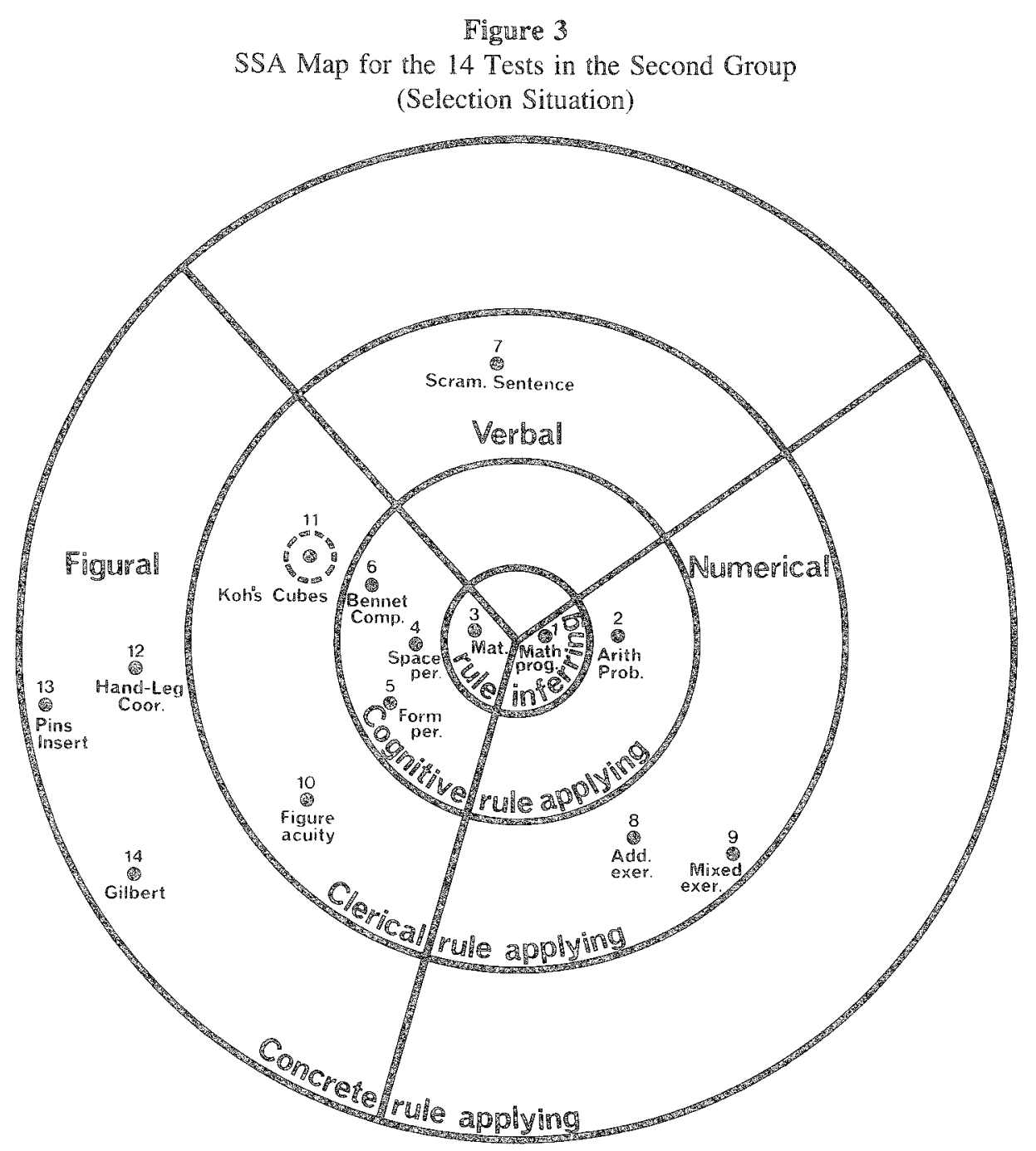

same structuple $a_{1} b_{1}$ in Figure 2. These are clearly closer to each other than to the Identifying Figures in Small Print test, which represents structuples $a_{3} b_{3}$.

Furthemore, both figures have a similar configurative structure, as is expected according to the mapping sentence: a radex configuration formed by joint action of the language presentation facet $(\mathbb{A})$, which divides the space radially (polarizing facet), and the mental operation facet $(B)$, which divides the space from the center to the outside in concentric rings (modulating facet). Certain implications are evident concerning the order of elements in Facet $\mathbb{B}$. This facet divides the space so that (1) the rule-inferring ability tests occupy the central region, (2) encircled contiguously by the cognitiveabstract rule applying test region, (3) next by the clerical rule-applying tests regions, and (4) furthest by the concrete rule-applying tests region. Hence, the tests in each region are much closer to each other than to tests outside the region. For instance, Mathematical Progressions tests and Raven Progressive Matrices tests are much closer to each other than to Addition Exercises tests (Figure 3), 
since both pertain to the rule-inference region, while the latter pertains to the clerical rule-applying region.

There is, however, one noticeable exception to the above conclusion: In general, the ability tests representing structuple $a_{3} b_{4}$ in Figure 3 cluster together in the figural area within the concrete rule circle. This is correct for all of them, except for Koh's cubes test, which is unexpectedly situated in the clerical circle. It may be reasonable to suppose that this seemingly odd finding could be accounted for by the fact that it presents stimuli in a concrete form, although the type of mental operation required by this test is the rule of inference. Thus, this test is positioned somewhere in the mid area, as is indeed shown by its place in Figure 3 .

\section{Discerssion}

The implications of the results in this study are two-fold. First, although only a relatively small number of tests were involved, of which not all the structuples were equally represented (if at all) by tests, and although a new elaborated mental operation facet (instead of the original one generated by Schlesinger \& Guttman, 1969) was used, the radex proved again to be an appropriate configuration of the structure of ability tests. This conclusion is further supported by the fact that the radex structure did not evolve by chance, but rather that the empirical SSA results confirmed what had previously been predicted by the mapping sentence of the current study. Second, the configuration of points representing the ability tests exhibited the same structure (the radex) in both replications, albeit practically the tests administered to the testees in the two samples differed greatly, as dictated by the objective of testing (i.e., vocational guidance versus selection and placement). Consequently, it appears plausible to conclude that the radex structure can be expected to reproduce itself even if other tests are utilized, given that the two facetsmental operation and language of presentationare represented.

Of additional interest is the fact that those few tests employed in both replications (e.g., Raven's Progressive Matrices) retained location in the same regions of the radex in Figure 2 as in Figure 3 , thus indicating that the placement of a test in the radex is apparently invariant with respect to other tests in the same battery; rather, the location is predictable by the mapping sentence. In contrast, the structural invariance is clearly opposed to the salient difference in patterns of intercorrelation between tests, notably upon scrutiny of Tables 1 and 2. This probably demonstrates that intercorrelations are to a large extent affected by various extraneous factors (e.g., characteristics of examinees) unlike the remarkable stability of the configurative structure-the radex. In this regard the authors are, in essence, consistent with Elizur (1982), who arrived at a similar conclusion in his investigation of work values structure. In order to further support the conclusions concerning this structure, more studies across various sociodemographic characteristics of examinees (e.g., age, level of education, type of occupation) are certainly needed; they should, in addition, deal with the objectives of testing (e.g., assessment of managerial potential, assessment for promotion and for placement in organizations).

A further implication emerged from the findings concerning the radex structure. As mentioned previously, the mental operation facet divided the space into four concentric regions. These regions may be conceived as being arranged in a gradient order formed according to mental abstractness when the concrete rule-application is the lowest level and rule inference is the highest. This implies that a taxonomy of ability tests might be devised according to the above-mentioned four levels of abstractness. Further, this taxonomy would be maintained, no matter in what language test items are presented -verbal, numerical, or figural.

If the veracity of this conclusion is proved in further similar replications, a practical contribution, in addition to the theoretical contribution, could be made to the understanding of the underlying structure of ability tests. For instance, in order to design a battery of ability tests for selection, it would only be necessary to obtain informationvia a job analysis procedure-about the mental operation and the language of presentation that predominantly accounts for successful performance in 
that job. Then, all that would be required would be to select or to construct tests corresponding to the type of mental operation and the chosen language of presentation revealed in the job analysis process.

Some readers may consider this suggestion to be oversimplified. The authors trust, however, that as it is theoretically reasonable, it offers an approach for devising test batteries from a minimal number of components that would still contain all the relevant core elements. Some redundancy among predictors would exist, even if the proposed test battery procedure is followed, since, as has been pointed out, the tests tend to relate to one another. Yet, this redundancy would be substantially smaller than that expected in a test battery structured in a different manner. Some overlap among predictors, however, would not be detrimental as long as it is not too large and it increases the overall validity of the test battery as a whole (cf. Tziner \& Dolan, 1982).

To conclude, the authors agree with those researchers who maintain that the ultimate test for the validity of findings is their recurrence in mumerous studies (cf. James, Mulaik, \& Brett, 1982). For this reason additional research is suggested in order to accurately assess the extent of recurrence of the findings, in this study. This is particularly the case when comparing results from facet analysis, employed here with those emanating from other methods of structure exposure, such as Kruskal's multidimensional scaling analysis (Kruskal \& Wish, 1978).

\section{Reanerences}

Brainerd, C. J. (1978). Piaget's theory of intelligence. Englewood Cliffs NJ: Prentice-Hall.

Cunningham, W. R. (1981). Ability factor structure differences in adulthood and old age. Multivariate Behavioral Research, 16, 3-22.

Elizur, D. (1981). The importance and structure of job outcomes. Unpublished manuscript, Bar-Ilan University.

Elizur, D. (1982). Facets of work values: A structural analysis of work outcomes. Unpublished manuscript, Bar-Ilan University.

Gratch, H. (1973). Twenty-five years of social research:
The story of the Israel Institute of Applied Social Research. Jerusalem: Jerusalem Academic Press.

Guilford, I. P. (1980). Fluid and crystallized intelligences: Two Panciful concepts. Psychological Bulletin, 88, 406-412.

Guilford, J. P. (1981). Higher-order structure-of-intellect abilities. Multivariate Behavioral Research, 16 , $411-435$.

Guttman, L. (1958). What lies ahead for factor analysis? Educational and Psychological Measurement, 18,497515.

Gutman, L. (1968). A general nonmetric technique for finding the smallest coordinate space for configuration of points. Psychometrika, 33, 465-506.

James, L. R., Mulaik, S. A., \& Brett, J. M. (1982). Conditions for confirmatory analysis and causal inference. San Francisco: Sage Publications.

Kelderman, H., Mellenbergh, G. I., \& Elshout, I. J. (1981). Guilford's facet theory of intelligence: An empirical comparison of models. Mulvivariate Rehavioral Research, 16, 37-61.

Kenny, C., \& Canter, D. (1981). A facet structure for nurses' evaluation of ward designs. Joumal of $O c$. cupational Psychology, 54, 93-108.

Kruskal, J. B., \& Wish, M. (1978). Multidimensional scaling. London/Beverly Hills CA: Sage Publications.

Lingoes, J. C. (1973). The Guttman-Lingoes nonmetric program series. Ann Arbor: Mathesis Press.

Piaget, J. (1956). The child's conception of space. London: Roufledge \& Kegan Paul.

Shapira, Z., \& Zevulun, E. (1979). On the use of facet analysis in organizational behavior research: Some concepiual considerations and an example. Organizational Behavior and Human Performance, 23,411 428.

Shirom, A. (1982). What is organizational stress? A facet analytic conceptualization. Joumal of Occupational Behavior, 3, 21-38.

Schlesinger, $\mathbb{I}$. M., \& Guttman, L. (1969). Smallest space analysis of intelligence and achievement tests. Psychological Bulletin, 71, 95-100.

Shye, S. (Ed.) (1978). Theory construction and data analysis in the behavioral sciences. San Francisco: Jossey-Bass.

Tziner, A., \& Dolan, $\mathbb{S}$. (1982). Validity of an assessment center for identifying future female officers in the military. Journal of Applied Psychology, 67, 728736.

\section{Acknowledgments}

Both authors contributed equally to this article. The authors thank $L$. Guttman for his helpful comments on an earlier droft of this arricle. 


\section{Author's Address}

Send requests for reprints or further information to Aharon Tziner, Department of Labor Studies, Faculty of Social Sciences, Tel Aviv University, Ramat Aviv, Tel Aviv 69978 , Israel.

Downloaded from the Digital Conservancy at the University of Minnesota, http://purl.umn.edu/93227.

May be reproduced with no cost by students and faculty for academic use. Non-academic reproduction requires payment of royalties through the Copyright Clearance Center, http://www.copyright.com/ 